\title{
Analysis of the orbital stability close to the binary asteroid (90) Antiope
}

\author{
S. Aljbaae ${ }^{1 \star}$, A. F. B. A. Prado ${ }^{1}$ D. M. Sanchez ${ }^{1}$, H. Hussmann ${ }^{2}$ \\ ${ }^{1}$ Division of Space Mechanics and Control, INPE, C.P. 515, 12227-310 São José dos Campos, SP, Brazil \\ 2 German Aerospace Center (DLR), Institute of Planetary Research, Berlin, Germany
}

Accepted ... . Received 2016 ...; in original form 2016 September 13.

\begin{abstract}
We provide a generalized discussion on the dynamics of a spacecraft around the equal-mass binary asteroid (90) Antiope, under the influence of solar radiation pressure at the perihelion and aphelion distances of the asteroid from the Sun. The polyhedral shape of the components of this asteroid is used to accurately model the gravitational field. Five unstable equilibrium points are determined and classified into 2 cases that allow classifying of the motion associated with the target as always unstable. The dynamical effects of the mass ratio of our binary system are investigated. We tested massless particles initially located at the periapsis distance on the equatorial plane of the primary of our binary asteroid. Bounded orbits around our system are not found for the longitudes $\lambda \in$ $\{60,90,120,240,270,300\}$. We also discuss the orbital dynamics in the full potential field of (90) Antiope. The tested motions are mainly dominated by the binary's gravitational field, No significant effects of the SRP are detected. For $\lambda=180^{\circ}$, less perturbed orbits are identified between 420 and $700 \mathrm{~km}$ from the centre of the system, that corresponds to orbits with $\Delta a<30 \mathrm{~km}$ and $\Delta e<0.15$. All the orbits with initial periapsis distance smaller than $350 \mathrm{~km}$ either collide with components of our asteroid or escaped from the system.
\end{abstract}

Key words: Celestial mechanics - gravitation âĂŞ Minor planets âĂŞ asteroids: individual: (90) Antiope.

\section{INTRODUCTION}

The prospect of a mission to investigate multiple asteroid systems is of particular interest for our understanding of the solar system history and for measuring quantities that are difficult to obtain, such as masses and densities as well as thermal, mechanical, and interior properties (Margot et al. 2015). Several multiple asteroids have been found in the Solar System, none of them has yet been visited by a spacecraft. However, NASA proposed a mission called DART (Double Asteroid Redirection Test) to Didymoon, the smaller body of the Potentially Hazardous binary asteroid (65803) Didymos. This sub-kilometre synchronous binary system is a near-Earth object with a mass-ratio of 0.01 (Michel et al. 2018; Cheng et al. 2018). Its orbit is classified as an Apollo group by JPL's Small-Body Database Browser ${ }^{1}$ and an Amor group by IAU Minor Planet Center ${ }^{2}$. In fact, many of the observed binaries in the solar system possess significantly larger mass ratios than Sun-planet or planet-moon systems. Several efforts

\footnotetext{
* safwan.aljbaae@gmail.com

1 https://ssd.jpl.nasa.gov/sbdb.cgi?sstr $=2065803$

2 https://www.minorplanetcenter.net/db_search/show_object?

object_id $=65803$
}

have already been focused on the dynamics near binary systems. For example, Benest $(1976,1974)$ studied the effects of the mass ratio on the existence of retrograde satellites in the circular plane. Gabern, Koon \& E. (2005) presented a simple model to describe the motion of a massless particle around two point masses. Scheeres (2007) studied the dynamics of contact binary asteroids, describing the minimum energy configurations as a function of the total angular momentum. Hussmann et al. (2012) investigated the stability of orbits around the MarcoPolo-R target asteroids, the binary asteroid 175706 (1996 FG3), with complex gravity fields perturbed by solar radiation pressure. The authors modelled the gravity field as a homogeneous tri-axial ellipsoidal harmonic expansion up to fourth-degree and order. Bosanac, Howell \& Fischbach (2015a,b) explored the dynamical structure near a large mass ratio binary system using the circular restricted three-body problem. Woo (2014) studied the motion of a spacecraft in the vicinity of a binary asteroid system. The author considered the inertia properties of the irregularly shaped bodies to calculate the gravitational potential of the system. The motion on the binary system was considered as a planar full two-body problem. The Solar Radiation Pressure (SRP hereafter) was not discussed in that work. Feng et al. (2016) analysed the general orbital 
motion around a contact binary asteroid system, based on the physical parameters of 1996 HW1. The author used a shape model consisting of two lobes (an ellipsoidal component and a spherical component). Dell'Elce et al. (2017) investigated the dynamical environment in the vicinity of the (65803) Didymos system. The authors used a quite simple model of cannonball SRP, where the shape of the satellite is approximated by a sphere and the SRP acceleration is considered always in the satellite-Sun direction. Jiang (2018) simulated the orbit for the moonlet in the potential of the primary of the binary asteroid 41 Daphne. Shi, Wang \& Xu (2018) presented a numerical method to search periodic orbits close to the binary asteroid (66391) 1999 KW4 without considering the SRP. Jean et al. (2018) used a fourth-order gravitational potential model to study the impact of a simple SRP flat plate model on a spacecraft around a binary system with a mass ratio parameter of 0.008585 . However, more realistic SRP models have also been investigated in a different environment. Xin, Scheeres \& X. (2016) studied the forced periodic motions caused by SRP around uniformly rotating asteroids. In this work, we explore the dynamical structure near a large mass ratio asteroid binary system under the influence of the SRP as presented in Xin, Scheeres \& X. (2016). We choose the binary asteroid (90) Antiope for numerical study.

Among the known binary asteroids in our Solar System, the (90) Antiope system is unique. With almost equalsize components (named Alpha and Beta in this work), separated by only $176 \pm 4 \mathrm{~km}$. It was the first doubly synchronous system discovered with ground-based observations using adaptive optics. Both components have $16.505046 \pm$ $0.000005 \mathrm{~h}$ periods synchronized to their mutual orbit period, most likely due to rapid tidal evolution (Taylor 2009; Taylor \& Margot 2010, 2011). That leads to estimate the total mass of the system to be $9.14 \pm 0.62 \times 10^{17} \mathrm{~kg}$ with a mean density of $1.67 \pm 0.23 \mathrm{~g} \mathrm{~cm}^{-3}$ (Merline et al. 2000; Bartczak et al. 2014). The origin of the (90) Antiope system is still not fully clear. Descamps et al. (2007) suggested that the system could have originated in a catastrophic collision in the Themis family probably during or just after the disruption of a 400-km parent asteroid about 2.5 Gyr ago. (90) Antiope is located in the outer asteroid belt, with a perihelion of $2.639 \mathrm{au}$ and an aphelion distance of $3.670 \mathrm{au}$. Its eccentricity $(0.166)$ is moderate, and its inclination with respect to the ecliptical plane is quite small $\left(2.207^{\circ}\right)$. The orbital period around the Sun is about $5.59 \mathrm{yr}$.

In this paper, we focus on the aspects of orbital dynamics around the binary asteroid (90) Antiope, considering the shape of both components taking into account the SRP. We explore the dynamical effects of the tidal dissipation that occurs between Beta and the deformation of Alpha due to Beta. The outline of our study is as follows. The physical properties of the polyhedral shape of the asteroid are presented in Sec. 2 of this article. In Sec. 3, we study the dynamical properties around the target calculating the Jacobi integral and the zero-velocity surfaces and the equilibrium points of the system. A general presentation of our numerical analysis of the stability of a massless particle around (90) Antiope is presented in Sec. 4 and 5. Our final comments and general observations are presented in Sec. 6 .

\section{PHYSICAL PROPERTIES FROM THE POLYHEDRAL SHAPE}

Using photometric observations, a non-convex model of the (90) Antiope binary asteroid is derived in Bartczak et al. (2014) (fig. 1). The authors applied the Shaping Asteroids with Genetic Evolution (SAGE) modelling technique for non-convex shapes. This technique randomly mutates sphere-like shapes model parameters until the optical light curves are fitted.
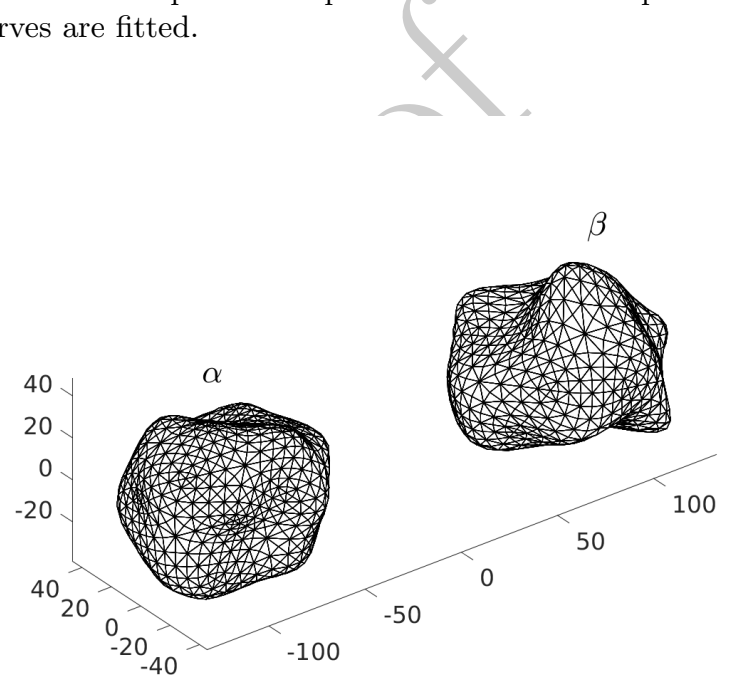

Figure 1. The (90) Antiope binary asteroid, using the shape model provided by Bartczak et al. (2014) with 1920 triangular faces for each component.

We used the algorithm of Mirtich (1996) to compute the $3 \mathrm{D}$ inertia tensor of the polyhedron shape of Alpha and Beta. We found that each body is perfectly oriented along its principal axes of inertia. This implies that the shape can safely be used to accurately study the orbital dynamics around the asteroid. We then used the algorithm of Werner (1997) to calculate the spherical harmonic coefficients of each body. In table 1 we present the main physical properties of each one of the components of the target, considering a homogeneous structure with uniform density of $1.67 \pm 0.23 \mathrm{~g} . \mathrm{cm}^{-3}$ (Bartczak et al. 2014). The shape of Alpha and Beta viewed in various perspectives is presented in Fig. 2.

Table 1. The Physical properties of the components of the 90 Antiope binary asteroid

\begin{tabular}{crr}
\hline & \multicolumn{1}{c}{ Alpha } & \multicolumn{1}{c}{ Beta } \\
\hline Effective diameters $(\mathrm{km})$ & 80.700619 & 80.285799 \\
Polyhedral volumes $\left(\times 10^{6} \mathrm{~km}^{3}\right)$ & 0.275188 & 0.270966 \\
Masses estimation $\left(\times 10^{17} \mathrm{~kg}\right)$ & 4.595642 & 4.525132 \\
Dynamical polar flattening: $J_{2}\left(-C_{20}\right)$ & 0.087493 & 0.101582 \\
Dynamical equatorial flattening $\left(C_{22}\right)$ & 0.012232 & 0.012214 \\
Moments of inertia $I_{x x} / M\left(\mathrm{~km}^{2}\right)$ & 601.746920 & 605.884328 \\
Moments of inertia $I_{y y} / M\left(\mathrm{~km}^{2}\right)$ & 680.648899 & 683.839847 \\
Moments of inertia $I_{z z} / M\left(\mathrm{~km}^{2}\right)$ & 782.287966 & 806.949293 \\
\hline & $a=46.400$ & $a=47.035$ \\
Equivalent ellipsoid $(\mathrm{km})$ & $b=41.934$ & $b=42.691$ \\
& $c=35.359$ & $c=34.741$ \\
\hline
\end{tabular}


The primary body $(\alpha)$
The secondary body $(\beta)$
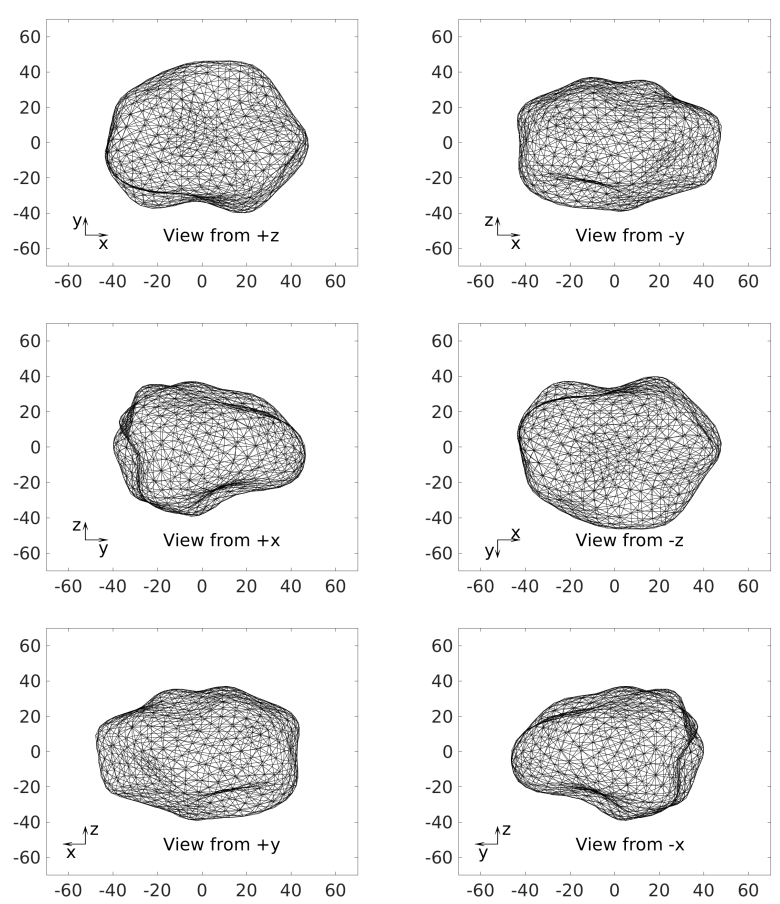

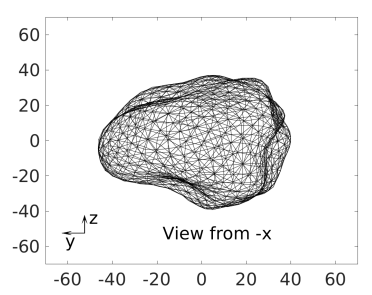

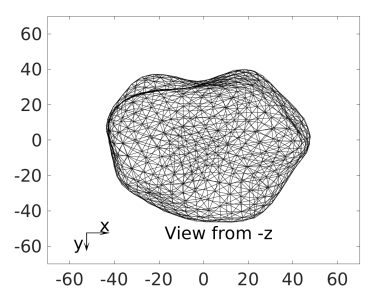
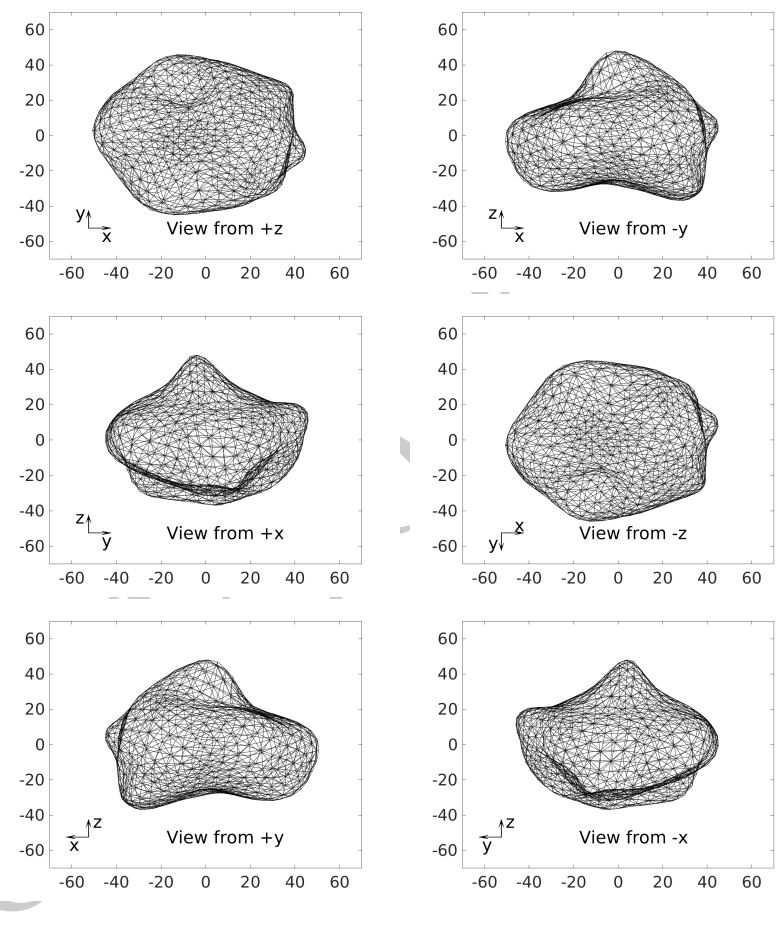

Figure 2. The polyhedral shape of the components of the binary asteroid (90) Antiope, shown in 6 perspective views $( \pm \mathrm{x}$, $\pm \mathrm{y}$, and $\pm \mathrm{z}$ ).

\section{EQUILIBRIUM POINTS}

To derive the equilibria of (90) Antiope, we consider the central body as: a) two points of mass b) two irregular polyhedral shapes. We found 5 equilibrium points, three of them are on the $\mathrm{x}$-axis (long axis) of the body-fixed frame (Fig. $3)$. The exact locations of these points and their Jacobi constant are listed in Table 2. We used the Mascon approach of Chanut, Aljbaae \& Carruba (2015) to include the irregular shape of the components of the asteroid (Alpha and Beta). We refer also to Venditti (2013) and Aljbaae et al. (2017) for more details about this approach. Considering or not the irregular shape of (90) Antiope, we observe that the positions of the equilibrium points $\left(E_{1}, E_{2}, E_{3}, E_{4}\right.$ and $\left.E_{5}\right)$ are moved by up to $0.833,0.735,1.007,0.226$, and 0.2100 $\mathrm{km}$, respectively. Analyzing the eigenvalues of the linearized system as described in Jiang et al. (2014) provides details on the stability of the equilibria. Table 3 shows the 6 eigenvalues for each equilibrium point. We notice that the irregular shape of the components of the central body did not change the stability of its equilibrium points. The points $\left(E_{1}, E_{2}\right.$, and $\left.E_{3}\right)$ are unstable and belong to the Case 2 as defined in Jiang et al. (2014) and Wang, Jiang \& Gong (2014), whereas the points $\left(E_{4}\right.$ and $\left.E_{5}\right)$ are unstable and belong to Case 5. That enables us to classify (90) Antiope as a Type II asteroid (Scheeres 1994). Thus, the motion associated with a near synchronous orbit is always unstable. However, we will investigate the orbital dynamics close to the target in the next section.
Table 2. Locations of the 5 equilibrium points of Antiope and their Jacobi constants $\mathrm{C}$ (using the shape model with 962 vertices).

\begin{tabular}{rrrrc}
\hline & \multicolumn{1}{c}{$\mathrm{x}(\mathrm{km})$} & $\mathrm{y}(\mathrm{km})$ & $\mathrm{z}(\mathrm{km})$ & $C\left(\mathrm{~km}^{2} \mathrm{~s}^{-2}\right)$ \\
\hline$E_{1}$ & 210.02581296 & -0.00000000 & -0.00000000 & $-0.59637110 \times 10^{-3}$ \\
$E_{2}$ & -0.64163063 & -0.00000000 & -0.00000000 & $-0.69571446 \times 10^{-3}$ \\
$E_{3}$ & -212.12157095 & -0.00000000 & -0.00000000 & $-0.60340428 \times 10^{-3}$ \\
$E_{4}$ & 1.24261377 & 152.69474870 & -0.00000000 & $-0.47758217 \times 10^{-3}$ \\
$E_{5}$ & 1.24261377 & -152.69474870 & -0.00000000 & $-0.47758217 \times 10^{-3}$ \\
\hline \multicolumn{5}{c}{ polyhedral shape of Alpha and Beta } \\
\hline$E_{1}$ & 210.65027715 & 0.21807567 & -0.50683747 & $-0.34813444 \times 10^{-3}$ \\
$E_{2}$ & -1.02371744 & 0.09124172 & -0.62070163 & $-0.70548286 \times 10^{-3}$ \\
$E_{3}$ & -212.90409408 & -0.63333825 & -0.00092261 & $-0.35005532 \times 10^{-3}$ \\
$E_{4}$ & 1.45436794 & 152.77330475 & 0.01514038 & $-0.34556327 \times 10^{-3}$ \\
$E_{5}$ & 0.98973277 & -152.74026816 & 0.03540602 & $-0.34553335 \times 10^{-3}$ \\
\hline \multicolumn{5}{c}{}
\end{tabular}

Table 3. Eigenvalues of the coefficient matrix of the 5 external equilibrium points

\begin{tabular}{rrrrrr}
\hline eigenvalues & \multicolumn{1}{c}{$E_{1}$} & $E_{2}$ & $E_{3}$ & $E_{4}$ & \multicolumn{1}{c}{$E_{5}$} \\
\hline \multicolumn{7}{c}{ Mascon model, uniform density } \\
\hline \multicolumn{7}{c}{ Two point masses } \\
\hline \multicolumn{7}{c}{$10^{\times-4}$} & \multicolumn{1}{c}{$\times 10^{\times-4}$} \\
\hline$\lambda_{1}$ & -1.2114 & -4.0130 & -1.2295 & $-0.6677+1.0024 i$ & $-0.6677+1.0024 i$ \\
$\lambda_{2}$ & +1.2114 & +4.0130 & +1.2295 & $-0.6677-1.0024 i$ & $-0.6677-1.0024 i$ \\
$\lambda_{3}$ & $+1.4003 i$ & $+3.0571 i$ & $+1.4086 i$ & $+0.6677+1.0024 i$ & $+0.6677+1.0024 i$ \\
$\lambda_{4}$ & $-1.4003 i$ & $-3.0571 i$ & $-1.4086 i$ & $+0.6677-1.0024 i$ & $+0.6677-1.0024 i$ \\
$\lambda_{5}$ & $+1.3203 i$ & $+2.9991 i$ & $+1.3281 i$ & $+1.0574 i$ & $+1.0574 i$ \\
$\lambda_{6}$ & $-1.3203 i$ & $-2.9991 i$ & $-1.3281 i$ & $-1.0574 i$ & $-1.0574 i$ \\
\hline \multicolumn{7}{c}{ polyhedral shape of Alpha and Beta } \\
\hline$\lambda_{1}$ & -1.2300 & -4.3002 & -1.2563 & $-0.6715+1.0009 i$ & $-0.6691+1.0004 i$ \\
$\lambda_{2}$ & +1.2300 & +4.3002 & +1.2563 & $-0.6715-1.0009 i$ & $-0.6691-1.0004 i$ \\
$\lambda_{3}$ & $+1.4026 i$ & $+3.2437 i$ & $+1.4154 i$ & $+0.6715+1.0009 i$ & $+0.6691+1.0004 i$ \\
$\lambda_{4}$ & $-1.4026 i$ & $-3.2437 i$ & $-1.4154 i$ & $+0.6715-1.0009 i$ & $+0.6691-1.0004 i$ \\
$\lambda_{5}$ & $+1.3349 i$ & $+3.1947 i$ & $+1.3458 i$ & $+1.0652 i$ & $+1.0631 i$ \\
$\lambda_{6}$ & $-1.3349 i$ & $-3.1947 i$ & $-1.3458 i$ & $-1.0652 i$ & $-1.0631 i$ \\
\hline
\end{tabular}



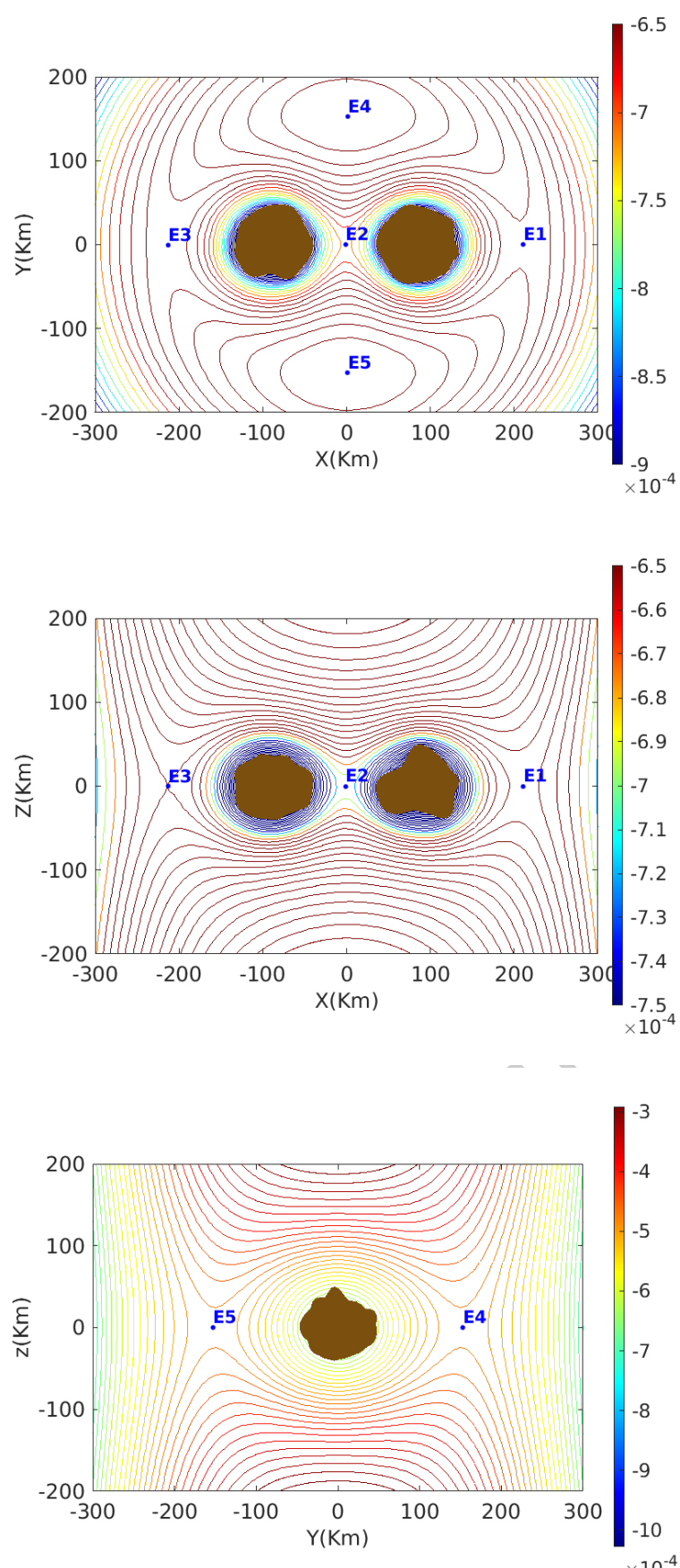

Figure 3. Zero-velocity curves and equilibrium points of (90) Antiope in the xoy, xoz and yoz planes, using the nonconvex shape model. The colour code gives the value of the Jacobi constant in $\mathrm{km}^{2} \mathrm{~s}^{2}$.

\section{EQUATIONS OF MOTION}

Our aim in this section is to investigate the dynamical environment in the full gravitational potential of the binary asteroid (90) Antiope, taking into account the 3D irregular shape of both components. We will concentrate on one of the components (Alpha) and consider the case of a spacecraft inside the Hill's sphere of the asteroid, that varies from $21,122.519$ to $29,377.107 \mathrm{~km}$ at perihelion and aphelion, respectively. We choose to work on an OSIRIS-REx-like spacecraft, as characterized in Scheeres, Sutter \& Rosengren (2013). Our dynamics takes place significantly far from any other celestial body, where the motion is dominated by the gravitational field of the binary target, that allows us to safely neglect the solar gravity perturbation and any perturbations from the remaining bodies in the Solar System. We use the Bulirsch-Stoer variable step-size algorithm (Bulirsch \& Stoer 1966), optimized for accuracy of $10^{-12}$, covering 100 days. In our model, the motions of Beta and the spacecraft around Alpha are integrated with the classical equations of motion in the body-fixed frame of reference. The mass of the spacecraft is neglected, therefore, no effects of the spacecraft on Beta exist. We classified the orbit types into (a) bounded motion: the test body stays inside the system's disk with a radius chosen to be $10,000 \mathrm{~km}$. We considered a relatively high disk radius to be sure that the orbits beyond this limit will certainly escape from the system and not return back (b) escape motion: the semimajor axis of the orbit, in this case, will diverge when eccentricity $\geqslant 1$. (c) collision: a collision with Alpha and Beta occurs when the test body crosses the surface of the polyhedral shape of the components of the asteroid as we will see in Sec. 5.

As an initial effort to understanding the orbital structure close to the binary target, we search for bounded orbits around it neglecting the shape of Alpha or Beta. We used two different kinds of initial conditions. We first calculated the initial conditions using the time derivative of the position matrix given by Murray \& Dermott (1999), using the classical orbital parameters $(a, e, i, \varpi, w$, and $f)$. We tested planar prograde orbits, varying the initial semi-major axis $\left(a_{0}\right)$ in the interval $[200,900] \mathrm{km}$ with a step size of $10 \mathrm{~km}$. In fact, from the potential point of view, our binary system is seen as a point mass at its centre of masses beyond 900 $\mathrm{km}$, as shown in the top panel of Fig. 4, where we calculate the gravitational potential of (90) Antiope considering it as a single point mass located at the barycenter of the system $\left(\mathrm{U}_{\mathrm{cm}}\right)$, two-point masses $\left(\mathrm{U}_{\alpha}\right.$ and $\left.\mathrm{U}_{\beta}\right)$, or two polyhedral shapes $\left(\mathrm{U}_{\text {shape }}\right)$. The difference $\mathrm{U}_{\mathrm{cm}}-\left(\mathrm{U}_{\alpha}+\mathrm{U}_{\beta}\right)$ and $\mathrm{U}_{\text {shape }}-\left(\mathrm{U}_{\alpha}+\mathrm{U}_{\beta}\right)$ are presented in red and blue in the top panel, respectively. We can notice that the red and blue curves can hardly be distinguished. However, the relative error between these curves is presented in the bottom panel. The initial eccentricity is increasing in the interval $[0,0.9]$ with a step size of 0.1 . We changed the initial longitude of the periapsis $(\varpi)$ from $0^{\circ}$ to $330^{\circ}$ in intervals of $30^{\circ}$, while the initial values of the argument of pericenter $(w)$ and mean anomaly $(f)$ are fixed to 0.0 (initial $\mathrm{I}-1$, hereafter) then to $180^{\circ}$ (initial I-2). In other kinds of initial conditions (initial I-3), we initially located the spacecraft at the periapsis distance $\left(r_{0}\right)$ on the equatorial plane, like in Aljbaae et al. (2017, 2019). We also varied the initial periapsis between 250 and $900 \mathrm{~km}$ from the centre of Alpha with an interval of 10 $\mathrm{km}$. We considered that all the particles start at 11 different longitudes $(\lambda)$ distributed equally in the interval $[0,330]^{\circ}$. We also varied the initial eccentricity between 0 and 1 with an interval of 0.1 . In Fig. 5, we present the initial positions generated using the three sets of initial conditions.

We calculated all our initial conditions considering the existence of Alpha and Beta, as point of masses, by calculating the relative velocity of the particle. Bounded orbits 

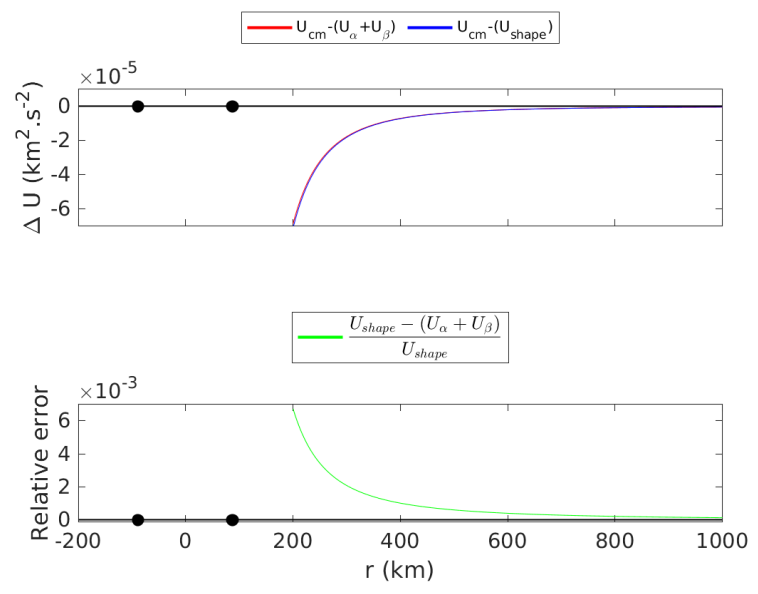

Figure 4. Top panel: The difference in the potential of the binary asteroid (90) Antiope, considering it as a single point mass located at the barycenter of the Antiope system $\left(\mathrm{U}_{\mathrm{cm}}\right)$, two-point masses $\left(\mathrm{U}_{\alpha}\right.$ and $\mathrm{U}_{\beta}$, blue), or two polyhedral shapes ( $\mathrm{U}_{\text {shape }}$, red). Bottom panel: the relative error between the potential estimated considering two point masses or two polyhedral shapes

around the binary system of Antiope are not found for $\varpi$ or $\lambda= \pm 90^{\circ} \pm 30^{\circ}$. For the sake of simplicity, we presented, in Fig. 6 , only the results for $\varpi$ and $\lambda=0^{\circ}$. We notice that more bounded orbits were found using the initial conditions I-2 and I-3. We also presented, in the last figure, the variation of the semi-major axis and eccentricity for each set of initial conditions. We can clearly see that our third group of initial conditions generate less perturbations on $a$ and $e$. Interested readers could find more information on results for orbits with other values of the initial $\varpi$ and $\lambda$ in appendix A. We notice that the dynamical systems around (90) Antiope are related to symmetry. Overall, for the rest of our work, we decided to consider the initial conditions I-3, limiting our analysis on the values of $\lambda=0,30,150,180,210,330^{\circ}$.

\subsection{Dynamical model}

Next, we define the generic dynamical model for the rest of this paper. We first calculated the total gravitational attraction exerted by Alpha on the spacecraft within 600 $\mathrm{km}$ from the central body, that reaches $8.563157 \times 10^{-8}$ $\mathrm{km} \mathrm{s}^{-2}$ considering the central body as a point of mass, $8.544278 \times 10^{-8} \mathrm{~km} \mathrm{~s}^{-2}$ using a spherical harmonics expansion up to order and degree four, as presented in Sanchez, Prado \& Yokoyama (2014); Sanchez \& Prado (2017), and $8.520744 \times 10^{-8} \mathrm{~km} \mathrm{~s}^{-2}$ considering the full gravitational potential of Alpha using its polyhedral shape. In fact, considering the polyhedral shape avoids the accuracy and convergence problems of the harmonics approach, that makes it favourable to use, especially near the central body, despite the greater computation time needed. In Fig. 7 , we present an orbit near the central body, Alpha. In the left upper panel, the gravitational potential of Alpha and Beta is generated by a point mass model. The spherical harmonics up to degree and order four is used to expand the
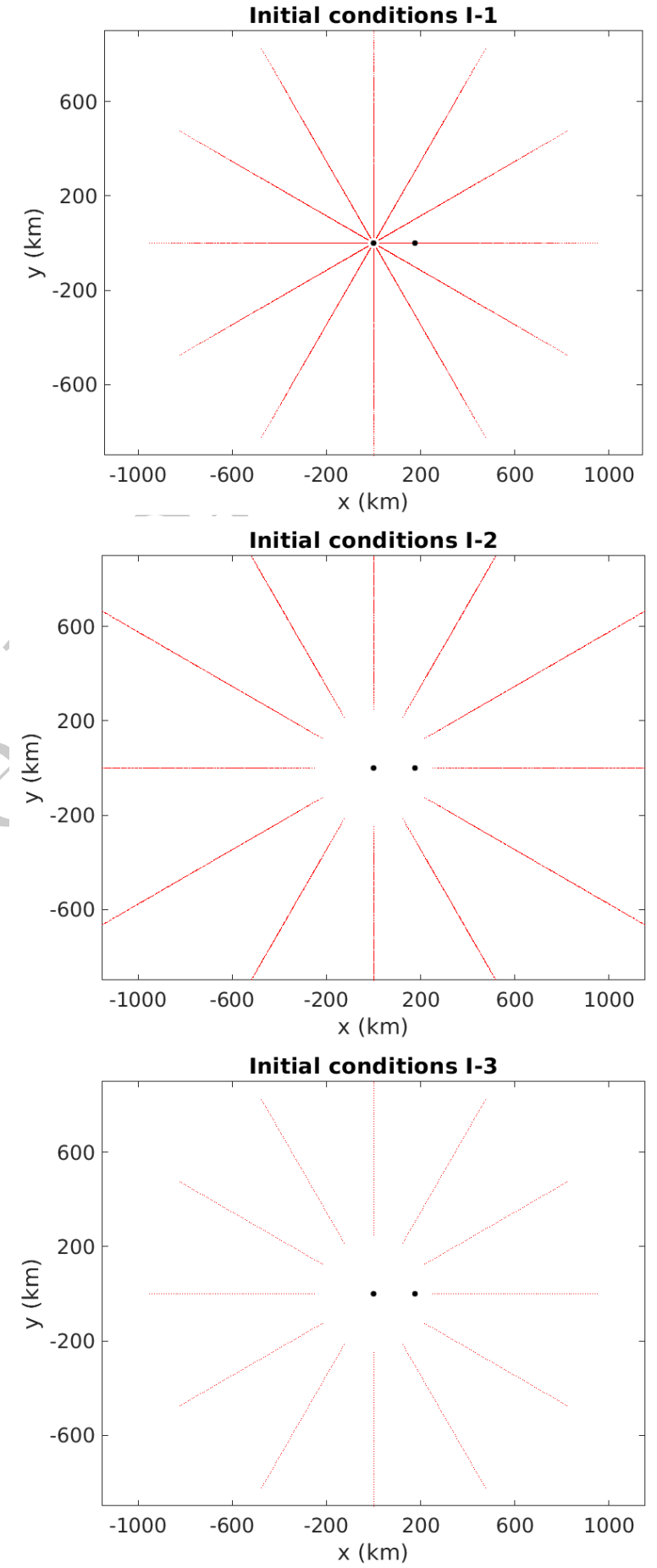

Figure 5. The initial positions of the simulated sample of particles around the binary asteroid (90) Antiope

gravitational potential of Alpha in the right upper panel. While we considered the polyhedral shape of Alpha in the left lower panel and the shape of Alpha and Beta in the right lower panel. One can clearly notice the effects of the polyhedral shapes of the components of our binary on the orbit. Thus, we think it is necessary to include the shape of both Alpha and Beta in the rest of this work according to 

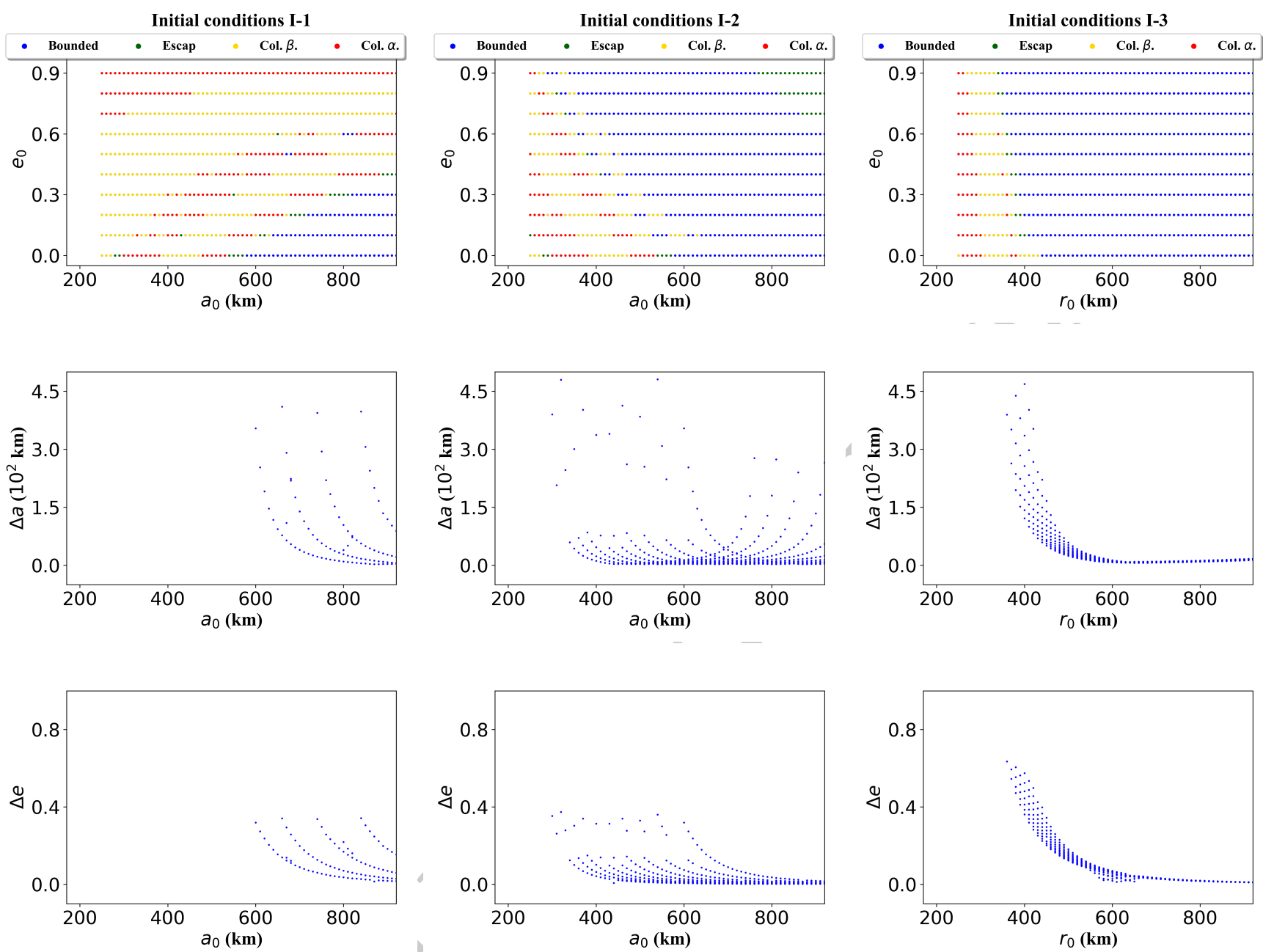

Figure 6. Effects of the initial conditions on planar prograde orbits close to (90) Antiope system, neglecting its polyhedral shape, after 100 days, considering $\varpi$ and $\lambda=0^{\circ}$. We recall that $a_{0}$ refers to the initial semimajor axis in (initial I-1 and I-2). While $r_{0}$ refers to the initial periapsis distance of the spacecraft in (initial I-3)

equations 1 and 2 .

We considered an OSIRIS-REx-like spacecraft, which means that the effective spacecraft mass-to-area ratio of 65 $\mathrm{kg} \mathrm{m}^{2}$ and a reflectance of 0.4. The total SRP acceleration can reach up to $8.016 \times 10^{-12} \mathrm{~km} \cdot \mathrm{s}^{-2}$ at perihelion or $4.144 \times 10^{-12} \mathrm{~km} . \mathrm{s}^{-2}$ at aphelion. The effect of the SRP at the perihelion and aphelion distances of the asteroid from the Sun is considered in this work, as described in Xin, Scheeres \& X. (2016). During the trial period of our integration, 100 days $(\sim 5.2 \%$ of the Antiope orbital period around the Sun), we consider the Sun moving in a circular orbit, with a constant latitude $\theta$ and time-varying longitude $\varphi=-\Omega t+\varphi_{0}$ (see Fig. 1 in Xin, Scheeres \& X. (2016)), $\Omega$ is the spin rate of the central body. The ecliptic latitude of the pole of our system was determined by Bartczak et al. (2014) to be $38^{\circ}$, which implies $\theta=38^{\circ}$. We will lie the initial direction of the Sun along the $\mathrm{x}$-axis $\left(\varphi_{0}=180^{\circ}\right)$. For more details on this force within our model, we refer the reader to Chanut et al. (2017). We also considered the effects of the shadowing phenomenon according to Xin, Scheeres \& X.
(2016). To demonstrate the effectiveness of our method to detect the shadows of Alpha and Beta, we show in Fig. 8 an orbit around Alpha fixing the position of the Sun along the $y$-axis and the position of Beta during the whole integration.

In fact, besides the familiar gravitational force between the masses, each body of our binary asteroid experiences an additional force arising from the non-spherical mass distribution. It is the binary companion's tidal dissipation, which is one of the secondary effects that disturb the gravity environment of an orbiting spacecraft. The distance between our binary components is $176 \mathrm{~km}$. This distance is beyond the classical Roche limit (between 58 up to $98 \mathrm{~km}$ ), below which tidal forces would tear the components apart. On long time scales, the orbits of Alpha and Beta are changing. This effect can be neglected for the duration of our mission (100 days). Tidal forces can also change the potential of the asteroids periodically. There might be a phase-lag of the response due to dissipation. However, for the double-synchronous state, this effect can also be neglected for the suite of this work.

Overall, the equations of motion that our model 
Point masses

Spherical harmonics

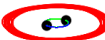

$\alpha$ Polyhedral shape

$\alpha$ and $\beta$ Polyhedral shape

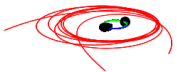

$r_{0}=300(\mathrm{~km})$

$e_{0}=0.5$

$i_{0}=0^{\circ}$

$\lambda_{0}=180^{\circ}$

Figure 7. Examples of orbits around the centre of mass over 100 days, using the initial conditions I-3. The point mass model is used in the left upper panel. The homogeneous spherical harmonics are considered in the right upper panel while the polyhedral shape of Alpha and Alpha/Beta are considered in the lower panels.

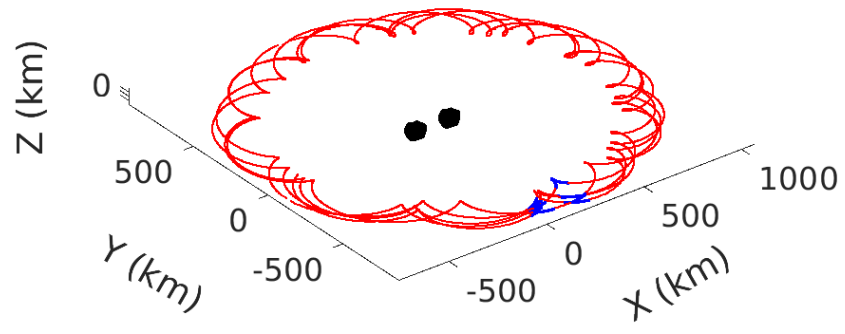

$r_{0}=600(\mathrm{~km}) \quad e_{0}=0.0 \quad i_{0}=0^{\circ} \quad \lambda_{0}=180^{\circ}$

Figure 8. An example of an orbit in the Alpha-fixed frame with the shadow of Alpha and Beta, using the initial conditions I-3, and fixing the position of the Sun along the y-axis and the position of Beta during the whole integration

describes have a spirit similar to that presented in Aljbaae et al. (2019). They are given by:

\section{The motion of Beta}

$$
\begin{aligned}
\ddot{x_{1}}-2 \Omega \dot{y_{1}} & =\Omega^{2} x_{1}+U_{x_{1}}+U_{x_{1}}^{\prime} \\
\ddot{y_{1}}+2 \Omega \dot{x_{1}} & =\Omega^{2} y_{1}+U_{y_{1}}+U_{y_{1}}^{\prime} \\
\ddot{z_{1}} & =U_{z_{1}}+U_{z_{1}}^{\prime}
\end{aligned}
$$

The motion of the spacecraft

$$
\begin{aligned}
\ddot{x_{2}}-2 \Omega \dot{y_{2}=} & \Omega^{2} x_{2}+U_{x_{2}}+U_{x_{2}}^{\prime}+U_{x_{1}}^{\prime}- \\
& \nu \frac{(1+\eta) \times 10^{8}}{B \times D^{2}} \cos \theta \cos \varphi \\
\ddot{y_{2}}+2 \Omega \dot{x_{2}}= & \Omega^{2} y_{2}+U_{y_{2}}+U_{y_{2}}^{\prime}+U_{y_{1}}^{\prime}- \\
& \nu \frac{(1+\eta) \times 10^{8}}{B \times D^{2}} \cos \theta \sin \varphi \\
\ddot{z_{2}}= & U_{z_{2}}+U_{z_{2}}^{\prime}+U_{z_{1}}^{\prime}- \\
& \nu \frac{(1+\eta) \times 10^{8}}{B \times D^{2}} \sin \theta
\end{aligned}
$$

where $\Omega$ is the spin rate of the primary. $U_{x}, U_{y}$ and $U_{z}$ are the first-order partial derivatives of the potential of the central body, calculated using the approach of Mascon 8 (Venditti 2013; Chanut, Aljbaae \& Carruba 2015; Aljbaae et al. 2017). $U_{x}^{\prime}, U_{y}^{\prime}$ and $U_{z}^{\prime}$ are the first-order partial derivatives of the secondary, calculated also by Mascon $8, \eta$ is the average total reflectance of the spacecraft, $B$ is the effective mass-to-area ratio of the spacecraft. In the following we take $\eta=0.4$ and $B=62 \mathrm{~kg} . \mathrm{m}^{-2} . D$ is the distance from the Sun in $\mathrm{km}$. We will consider the maximum and minimum SRP at the perihelion and aphelion distances. $\nu$ represent a factor to include the eclipse by an ellipsoidal asteroid taking the values 1 or 0, as defined in Xin, Scheeres \& X. (2016).

\section{STABILITY ANALYSIS}

In this section, we analyse equatorial, prograde orbits initially distributed around the real system $\alpha-\beta$ with the initial conditions I-3 given in Table 5 . We consider the perturbation due to the polyhedral shape of Alpha and Beta, and also the SRP at the perihelion and aphelion distances of the asteroid from the Sun. We notice that the SRP can significantly affect some individual orbits, as shown in Fig. 9. Our overall results at the perihelion are presented in Fig. 10. However, we notice that considering or not the SRP will not change the type of our orbits (Fig. 13 and 13 in Appendix B).

In accordance with section 4 , all the orbits started with initial longitudes $\lambda \in\{60,90,120,240,270,300\}$ either collide with the components of the asteroid or escaped to infinity. The Computational Geometry Algorithms Library $\left(\mathrm{CGAL}^{3}\right)$ is used in this section to handle collisions. CGAL provides an easy and efficient geometric algorithm accurate enough to find whether a point is inside or outside the polyhedron shape of our asteroid. In Fig. 11, we present all the orbits colliding with Alpha and Beta (left and right panel, respectively). We can see that not all the surface is accessible from equatorial prograde orbits. Collision speeds range depending on whether SRP is included is presented in Table 4. The SRP slightly increase the minimum speed.

The initial longitude $\lambda=180^{\circ}$ is the one that generates most of the bounded orbits in our system (Fig. 10). All the orbits with $r_{0}<350 \mathrm{~km}$ either collide with components of our asteroid or escaped from the system. To identify stable regions among the bounded orbits around (90) Antiope,

3 https://www.cgal.org/ 
Table 4. Collision speeds range $(\mathrm{m} / \mathrm{s})$ considering or not the SRP.

\begin{tabular}{c|cc|cc|cc}
\hline & \multicolumn{2}{|c|}{ without SRP } & \multicolumn{2}{c|}{ SRP at perihelion } & \multicolumn{2}{c|}{ SRP at aphelion } \\
& MIN & MAX & MIN & MAX & MIN & MAX \\
\hline$\alpha$ & 20.68 & 55.33 & 20.86 & 55.43 & 21.18 & 55.44 \\
$\beta$ & 23.24 & 54.21 & 24.47 & 54.22 & 24.75 & 54.22 \\
\hline
\end{tabular}

we checked the variation of the semi-major axes of the orbits around the centre of mass of our system. The minimum value of this variation is $16.2 \mathrm{~km}$ without considering the SRP while it becomes 15.2 and $17.7 \mathrm{~km}$ with the SRP at aphelion and perihelion distance, respectively. We found that the less perturbed orbits are located between $r_{0}=420$ and $r_{0}=700 \mathrm{~km}$. The variation of the semi-major axis $(\Delta a)$ and eccentricities $(\Delta e)$ in this area does not exceed $30 \mathrm{~km}$ and 0.15 , respectively. In Fig. 12 , we present our results. We notice that the SRP can slightly affect the considered stable area varying $\Delta e$ from 0.142 without the SRP to 0.134 and 0.137 at aphelion and perihelion, respectively.

\section{CONCLUSION}

In this paper we have presented a numerical analysis to explore the prograde motion of a massless test particle in the potential field of the equal-mass binary asteroid (90) Antiope, considering the shape of both components taking into account the SRP. We first obtained the physical properties of the polyhedral-shape of the components of our asteroid. The dynamical properties close to the target was investigated by calculating the zero-velocity surfaces and analyzing the stability of the equilibrium points of the system. We found that all the equilibrium points are unstable and the asteroid can be classified as a Type II according to Scheeres (1994). Using an OSIRIS-REx-like spacecraft, we investigated the orbital dynamics associated to (90) Antiope, neglecting the gravity perturbations from the Sun or other celestial bodies in the Solar System. We employed the Bulirsch-Stoer variable stepsize algorithm covering a period of 100 days and tested the effects of the mass ratio of our system on the planar orbits. Two different kinds of initial conditions were tested. In the first one, the initial state vector of the particles is calculated using the classical orbital parameters $(a, e, i, \varpi, w$, and $f$ ). In other kinds of initial conditions, we initially located the spacecraft at the periapsis distance on the equatorial plane of the central body, which generates less perturbed orbits compared to the first group of initial conditions. The binary companionâĂ Źs tidal dissipation was tested considering only the interaction between Beta and the deformation of Alpha due to Beta. We found a maximum value of the relative tidal effect on the distance spacecraft-Alpha less than $5 \times 10^{-5}$ that allows us to neglect this effect. Finally, we discuss the effects of the SRP on the orbital evolution close to (90) Antiope, we found that the dynamics in our selected region is dominated by the binary's gravitational field. However, the SRP can slightly affect scome orbits. In this work, we did not investigate the retrograde orbits. Usually, they tend to be more stable because they spend very little time in close proximity with the central body (close proximity happens very quickly), so gravitational perturbation is minimized. Finally, we can state that specific orbits between 420 and
$700 \mathrm{~km}$ from the centre of the system of (90) Antiope are allowing for good observations and characterizing the binary asteroid (90) Antiope.

\section{ACKNOWLEDGMENTS}

The authors would like to thank Dr. Przemyslaw Bartczak from the Astronomical Observatory of Adam Mickiewicz University in Poland for the fruitful discussions on the shape of the components of (90) Antiope. We would also like to thank the Coordination for the Improvement of Higher Education Personnel (CAPES), which supported this work via the grant $88887.374148 / 2019-00$.

\section{REFERENCES}

Aljbaae S., Chanut T. G. G., Carruba V., Souchay J., Prado A. F. B. A., Amarante A., 2017, MNRAS, 464, 3552

Aljbaae S., Chanut T. G. G., Prado A. F. B. A., Carruba V., Hussmann H., Souchay J., Sanchez D. M., 2019, MNRAS, 486, 2557

Bartczak P., Michałowski T., Santana-Ros T., Dudziński G., 2014, MNRAS, 443, 1802

Benest D., 1974, A\&A, 32, 39

Benest D., 1976, A\&A, 53, 231

Bosanac N., Howell K. C., Fischbach E., 2015a, Celestial Mechanics and Dynamical Astronomy, 122, 27

Bosanac N., Howell K. C., Fischbach E., 2015b, Celestial Mechanics and Dynamical Astronomy, 122, 27

Bulirsch R., Stoer J., 1966, Numerische Mathematik, 8, 1

Chanut T. G. G., Aljbaae S., Carruba V., 2015, MNRAS, 450, 3742

Chanut T. G. G., Aljbaae S., Prado A. F. B. A., Carruba V., 2017, MNRAS, 470, 2687

Cheng A. F. et al., 2018, Planet. Space Sci., 157, 104

Dell'Elce L., Baresi N., Naidu S. P., Benner L. A. M., Scheeres D. J., 2017, Advances in Space Research, 59, 1304

Descamps P. et al., 2007, Icarus, 187, 482

Feng J., Noomen R., Visser P., Yuan J., 2016, Advances in Space Research, 58, 387

Gabern F., Koon W. S., E. M. J., 2005, Conference Publications, 2005, 297

Hussmann H., Oberst J., Wickhusen K., Shi X., Damme F., Lüdicke F., Lupovka V., Bauer S., 2012, Planet. Space Sci., 70,102

Jean I., Misra A., Ng A., Dutta S., 2018, 2018 Space Flight Mechanics Meeting AIAA 2018-0956

Jiang Y., 2018, Results in Physics, 9, 1511

Jiang Y., Baoyin H., Li J., Li H., 2014, Ap\&SS, 349, 83

Margot J.-L., Pravec P., Taylor P., Carry B., Jacobson S., 2015, Asteroid Systems: Binaries, Triples, and Pairs, Michel P., DeMeo F. E., Bottke W. F., eds., pp. 355-374

Merline W. J., Close L. M., Dumas C., Shelton J. C., Menard F., Chapman C. R., Slater D. C., 2000, in Bulletin of the American Astronomical Society, Vol. 32, AAS/Division for Planetary Sciences Meeting Abstracts \#32, p. 1017

Michel P., Kueppers M., Sierks H., Carnelli e. a., 2018, Advances in Space Research, 62, 2261

Mirtich B., 1996, Journal of graphics tools, 1

Murray C. D., Dermott S. F., 1999, Solar system dynamics

Sanchez D. M., Prado A. F. B. A., 2017, AAS/AIAA Astrodynamics Specialist Conference, 162, 1507

Sanchez D. M., Prado A. F. B. A., Yokoyama T., 2014, Advances in Space Research, 54, 1008 

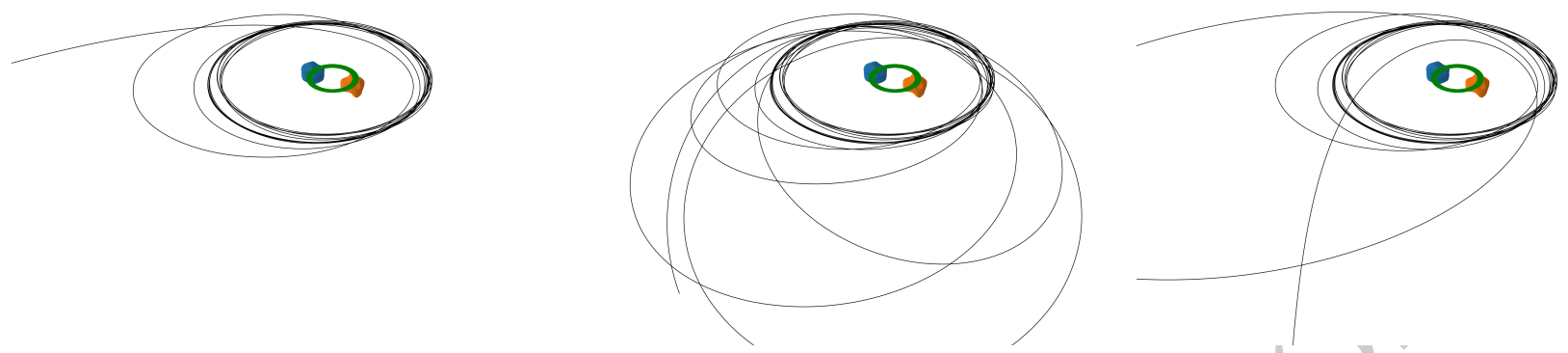

Figure 9. Example of equatorial orbit near the binary system of (90) Antiope over 100 days. In the left panel, we did not consider the SRP, while we consider this perturbation at the perihelion and aphelion distances from tyhe Sun in the centre and right panel, respectively.
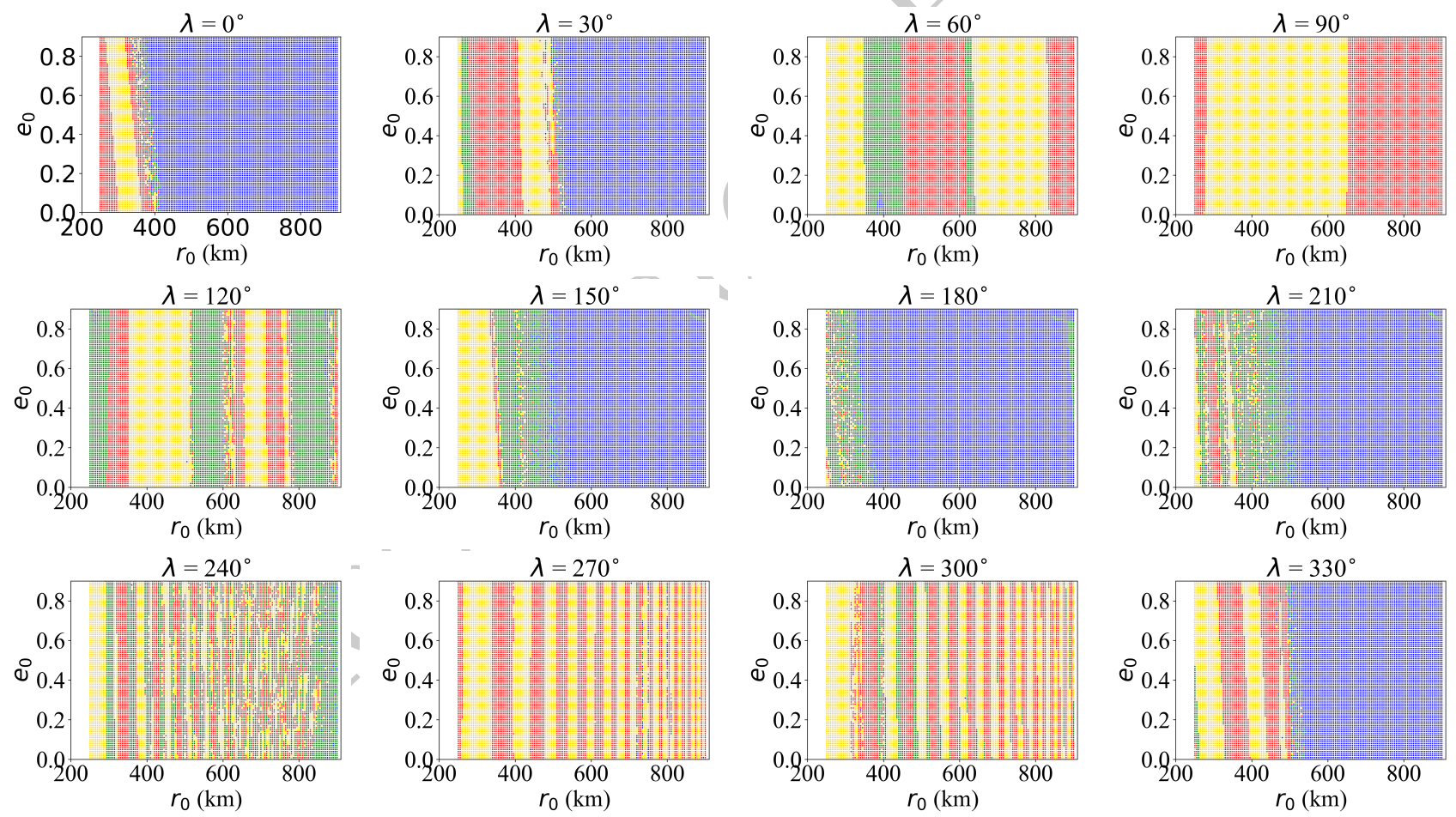

Figure 10. Type of orbits around the binary system of (90) Antiope at the perihelion distances from the Sun. The colour symbols are the same as in the top panel of Fig. 6

Scheeres D., Sutter B., Rosengren A., 2013, Advances in the Astronautical Sciences, 148, 3263

Scheeres D. J., 1994, Icarus, 110, 225

Scheeres D. J., 2007, Icarus, 189, 370

Shi Y., Wang Y., Xu S., 2018, Celestial Mechanics and Dynamical Astronomy, 130, 32

Taylor P. A., 2009, PhD thesis, Faculty of the Graduate School, Cornell University

Taylor P. A., Margot J.-L., 2010, Celestial Mechanics and Dynamical Astronomy, 108, 315

Taylor P. A., Margot J.-L., 2011, Icarus, 212, 661

Venditti F. C. F., 2013, PhD thesis, INPE, São José dos Campos

Wang X., Jiang Y., Gong S., 2014, Ap\&SS, 353, 105

Werner R. A., 1997, Computers and Geosciences, 23, 1071
Woo P., 2014, PhD thesis, Department of Mechanical Engineering, McGill University, Montreal, Quebec

Xin x., Scheeres D. J., X. H., 2016, Celest Mech Dyn Astr, 126, 405 


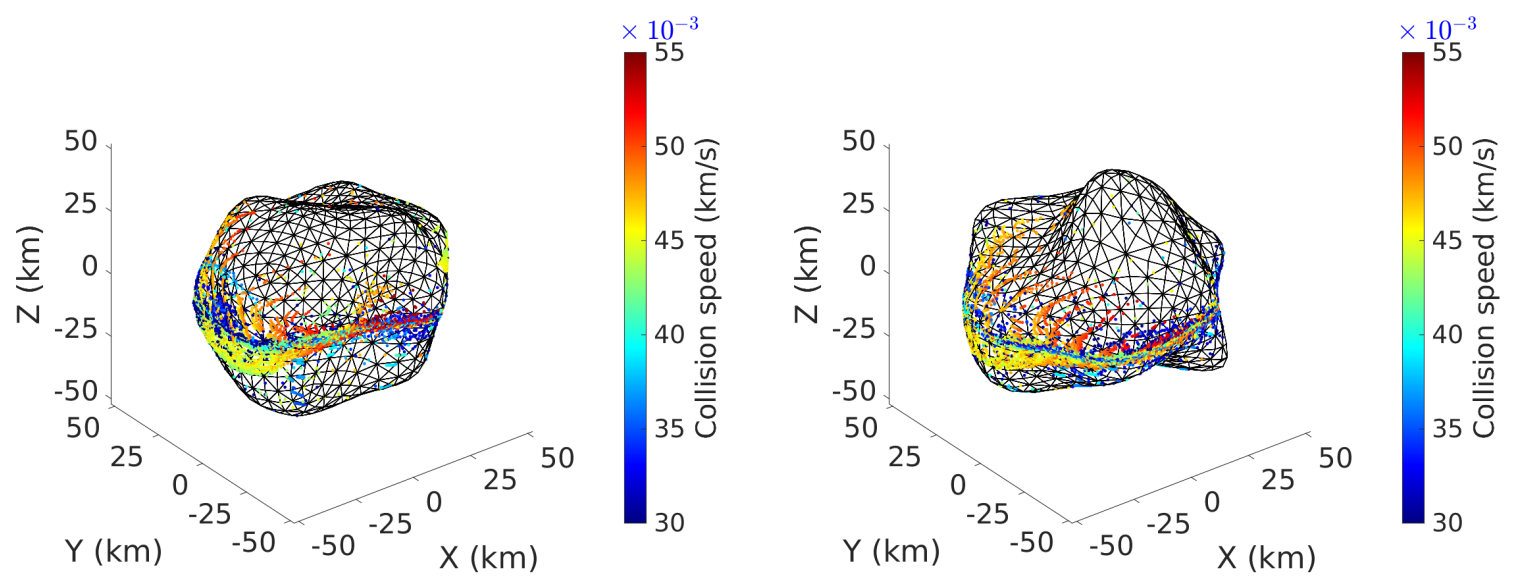

Figure 11. all the orbits colliding with Alpha (left panet) and Beta (right panet)
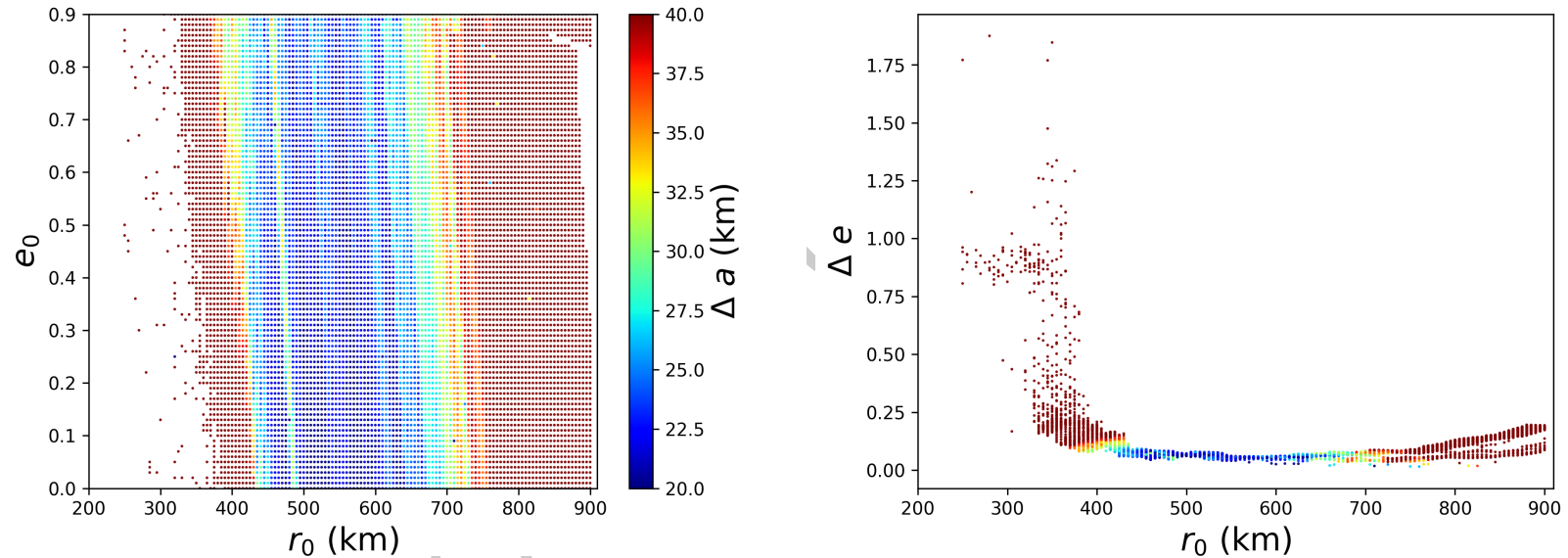

Figure 12. Variation of the semi-major axes and eccentricities of the bounded orbits around the centre of mass of Antiope system with initial longitude $\lambda=180^{\circ}$. 